\title{
The Science Case for PILOT I: Summary and Overview
}

\author{
J. S. Lawrence $\mathrm{A}, \mathrm{B}, \mathrm{AB}$, M. C. B. Ashley ${ }^{\mathrm{A}}$, J. Bailey ${ }^{\mathrm{A}}$, D. Barrado y Navascues ${ }^{\mathrm{C}}$, \\ T. R. Bedding ${ }^{\mathrm{D}}$, J. Bland-Hawthorn ${ }^{\mathrm{D}}$, I. Bond ${ }^{\mathrm{E}}$, F. Boulanger $^{\mathrm{F}}$, R. Bouwens ${ }^{\mathrm{G}}$,

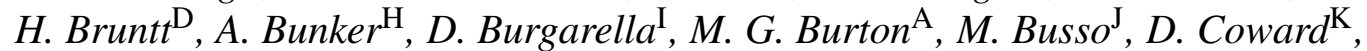

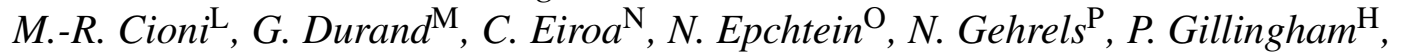 \\ K. Glazebrook $^{\mathrm{Q}}$, R. Haynes ${ }^{\mathrm{H}}$, L. Kiss ${ }^{\mathrm{D}}$, P. O. Lagage $^{\mathrm{M}}$, T. Le Bertre ${ }^{\mathrm{R}}$, C. Mackay ${ }^{\mathrm{S}}$,

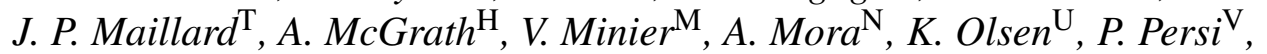 \\ K. Pimbblet ${ }^{\mathrm{W}}$, R. Quimby $^{\mathrm{X}}$,W. Saunders ${ }^{\mathrm{H}}$, B. Schmidt ${ }^{\mathrm{Y}}$, D. Stello ${ }^{\mathrm{D}}$, J. W. V. Storey ${ }^{\mathrm{A}}$, \\ C. Tinney ${ }^{\mathrm{A}}$, P. Tremblin ${ }^{\mathrm{M}}$, J. C. Wheeler ${ }^{\mathrm{Z}}$, and P. Yock ${ }^{\mathrm{AA}}$
}

A School of Physics, University of New South Wales, NSW 2052

B Present address: Department of Physics and Engineering, Macquarie University, NSW 2109 and Anglo-Australian Observatory, PO Box 296, Epping, NSW 1710

${ }^{\mathrm{C}}$ Laboratorio de Astrofísca Espacial y Física Fundamental (INTA), Madrid 28080, Spain

D Institute of Astronomy, School of Physics, University of Sydney, NSW 2006

E Massey University, Auckland 0745, New Zealand

F Institut d'Astrophysique Spatiale, Université Paris-Sud, Orsay 91405, France

G Department of Astronomy and Astrophysics, University of California Santa Cruz, Santa Cruz, CA 95064, USA

${ }^{\mathrm{H}}$ Anglo-Australian Observatory, NSW 1710

I Observatoire Astronomique de Marseille Provence, Université d'Aix-Marseille, Marseille 13388, France

${ }^{\mathrm{J}}$ Department of Physics, University of Perugia, Perugia 06123, Italy

${ }^{\mathrm{K}}$ School of Physics, University of Western Australia, Crawley, WA 6009

${ }^{\mathrm{L}}$ Centre for Astrophysics Research, University of Hertfordshire, Hatfield AL10 9AB, UK

${ }^{\mathrm{M}}$ Service d'Astrophysique, CEA Saclay, Saclay 91191, France

${ }^{\mathrm{N}}$ Departmento de Fisica Teórica C-XI, Universidad Autónoma de Madrid, Madrid 28049, Spain

${ }^{O}$ CNRS-Fizeau/UNSA, Nice 06108, France

P NASA/Goddard Space Flight Center, Greenbelt, MD 20771, USA

Q Centre for Astrophysics and Supercomputing, Swinburne University of Technology, Hawthorn, VIC 3122

R Observatoire de Paris, Paris 75014, France

${ }^{S}$ Institute of Astronomy, University of Cambridge, Cambridge CB3 OHE, UK

${ }^{\mathrm{T}}$ Institut d'Astrophysique de Paris, Paris 75014, France

U Kitt Peak National Observatory, National Optical Astronomy Observatory, Tucson, AZ 85719, USA

V Istituto Astrofisica Spaziale e Fisica Cosmica/INAF, Roma 00100, Italy

${ }^{\mathrm{W}}$ Department of Physics, University of Queensland, Brisbane, QLD 4072

X Astronomy Department, California Institute of Technology, Pasadena, CA 91125, USA

${ }^{Y}$ Research School of Astronomy and Astrophysics, Australian National University, ACT 2611

${ }^{\mathrm{Z}}$ Department of Astronomy, University of Texas, Austin, TX 78712, USA

AA University of Auckland, Auckland 1142, New Zealand

$\mathrm{AB}$ Corresponding author. Email: jsl@science.mq.edu.au

Received 2008 November 21, accepted 2009 May 13

\begin{abstract}
PILOT (the Pathfinder for an International Large Optical Telescope) is a proposed 2.5-m optical/ infrared telescope to be located at Dome $\mathrm{C}$ on the Antarctic plateau. Conditions at Dome $\mathrm{C}$ are known to be exceptional for astronomy. The seeing (above $\sim 30 \mathrm{~m}$ height), coherence time, and isoplanatic angle are all twice as good as at typical mid-latitude sites, while the water-vapour column, and the atmosphere and telescope thermal emission are all an order of magnitude better. These conditions enable a unique scientific capability for PILOT, which is addressed in this series of papers. The current paper presents an overview of the optical and instrumentation suite for PILOT and its expected performance, a summary of the key science
\end{abstract}


goals and observational approach for the facility, a discussion of the synergies between the science goals for PILOT and other telescopes, and a discussion of the future of Antarctic astronomy. Paper II and Paper III present details of the science projects divided, respectively, between the distant Universe (i.e. studies of first light, and the assembly and evolution of structure) and the nearby Universe (i.e. studies of Local Group galaxies, the Milky Way, and the Solar System).

Keywords: cosmology: observations - early universe — instrumentation: high angular resolution — site testing — stars: formation — telescopes

\section{Introduction}

The very low infrared background and atmospheric watervapour content of Antarctic plateau sites such as Dome C enable a telescope based there to achieve the sensitivity (at some wavelengths) of a telescope over three times that diameter located elsewhere (Lawrence 2004; Walden et al. 2005; Tomasi et al. 2006). The atmospheric turbulence above Dome $\mathrm{C}$ is also 2 to 3 times lower than that at even the best temperate sites (Lawrence et al. 2004; Agabi et al. 2006; Trinquet et al. 2008). An optical/infrared telescope at Dome $\mathrm{C}$ would thus be supremely powerful for its size, enjoying not only a substantial advantage in both sensitivity and photometric precision, but also having a wide-field, high-resolution, high-cadence imaging capability otherwise achievable only from space (Kenyon \& Storey 2006; Kenyon et al. 2006; Mosser \& Aristidi 2007).

PILOT (Pathfinder for an International Large Optical Telescope) is a key step to a major international observatory at Dome C. It is proposed as a high spatial resolution wide-field telescope with an optical design that is matched to the atmospheric conditions and a suite of instruments operating at wavelengths from the visible to the midinfrared and beyond (Saunders et al. 2008a,b). The specific aims for PILOT are:

- to perform cutting-edge science;

- to validate and further characterise the expected excellent natural seeing and the low thermal sky backgrounds at Dome $\mathrm{C}$, and to demonstrate that we can fully utilise these site conditions;

- to demonstrate that large optical/infrared telescopes can be built and operated in Antarctica within a reasonable time and cost.

The PILOT project, and its scientific motivation, has evolved over a number of years. Early discussions of the scientific potential of Antarctic plateau sites for generic optical and infrared telescopes were given by Burton et al. (1994) and Burton, Storey \& Ashley (2001). A more detailed investigation giving a broad range of potential science programs for a 2-m class Antarctic telescope was presented by Burton et al. (2005), based on a 'strawman' telescope and instrument suite configuration for PILOT. In the current series of papers, we present the next iteration of the PILOT science case. A series of leading-edge science drivers for the facility have been identified, and the specific observational and technological requirements have been defined for each. This science case has evolved in parallel with the telescope optical design and instrument suite configuration (see Saunders et al. 2008a,b) developed during the 'Phase A' PILOT design study ${ }^{1}$. It is the intention that the science cases and observing strategies described here, and the requirements that these make on the telescope and instrument design, will be further refined, iterated, and prioritised during the next phase of the PILOT project.

The key science objectives that have been identified for the PILOT project, grouped into seven themes, are:

1. First light in the Universe: to detect pair-instability supernovae and gamma-ray burst afterglows at high redshift. These objects represent the signatures of the final evolutionary stages of the first stars to form in the Universe.

2. The assembly of structure: to examine the properties of the first evolved galaxies to form in the Universe at high redshift, and to examine the assembly processes of galaxy clusters at moderate redshift.

3. Dark matter and dark energy: to probe the evolution of cosmological parameters via the observation of weak gravitationally-lensed galaxies and supernovae unaffected by dust extinction.

4. Stellar properties and populations: to increase our understanding of the formation and evolution of galaxies and stars, through investigations of the properties of stellar populations in nearby galaxies and stellar clusters.

5. Star and planet formation: to investigate the molecular phase of the Galaxy and explore the ecology of star formation, and to investigate the formation processes of stellar and planetary systems.

6. Exoplanet science: to directly detect 'free-floating' objects as low in mass as a few Jupiter masses, to firmly determine the abundance of ice-giant planets, and to characterise the atmospheric properties of a large number of hot Jupiters.

7. Solar system and space science: to investigate the atmospheric composition and dynamics of Venus and the atmospheric surface pressure of Mars, to observe coronal mass ejections from the Sun, and to obtain orbits for a large number of small-scale space debris (also known as 'space junk').

The depth and breadth of science proposed for the PILOT facility, despite its modest 2.5-m aperture, is indicative of the potential of the Dome $\mathrm{C}$ site. These same characteristics will also benefit future, larger telescopes,

\footnotetext{
${ }^{1}$ See http://www.phys.unsw.edu.au/pilot/.
} 


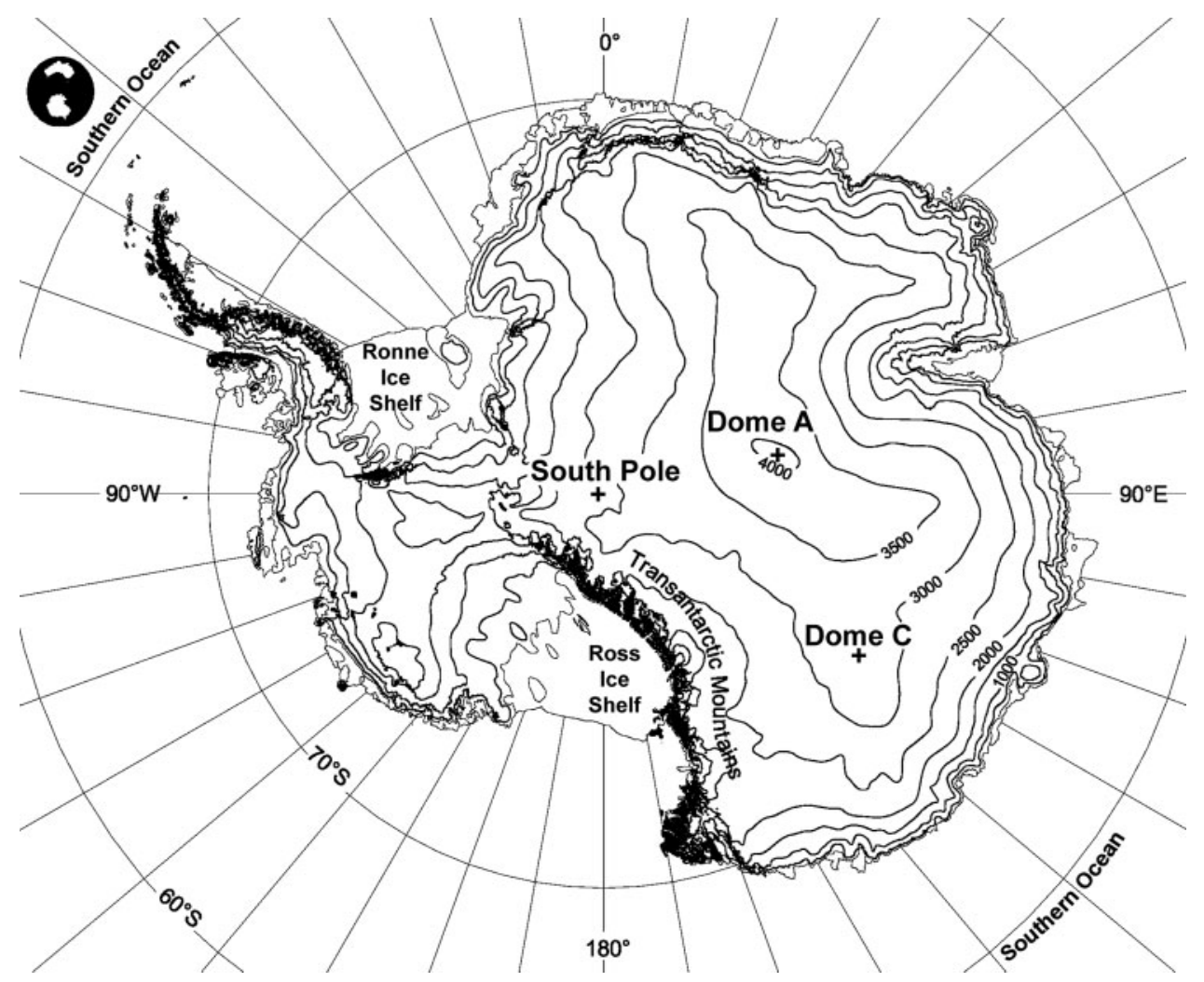

Figure 1 Map of Antarctica showing the high plateau stations South Pole, Dome C, and Dome A. Basic map courtesy of the Australian Antarctic Data Centre.

for which PILOT will act as a pathfinder. For example, an 8- to 10-m class Antarctic telescope would be extraordinarily powerful for very high resolution optical imaging over small fields and/or deep infrared imaging over extremely wide fields, enabling direct imaging of exoplanets and detailed investigations of the first stars and galaxies to form in the Universe.

This paper is organised as follows: Section 2 gives an overview of the Dome $\mathrm{C}$ site conditions, the PILOT optical design and instrumentation suite, and its expected performance. An analysis of the PILOT parameter space compared to current and future facilities is also given. Section 3 summarises, and provides the context for, the key PILOT science projects. A discussion of observing strategies for the facility is given in Section 4. Synergies between PILOT and other facilities, in particular the Giant Magellan Telescope, the Australian Square Kilometre Array Pathfinder, the Murchison Widefield Array, and the South Pole Telescope, are addressed in Section 5. Finally, Section 6 presents a vision for the future of Antarctic astronomy via a discussion of potential larger-scale astronomical facilities to follow PILOT. Paper II (Lawrence et al. 2009a) in this series presents a range of science projects for the PILOT facility that are aimed at observing and understanding the distant Universe (i.e. science themes 1-3 from the above list). Paper III (Lawrence et al. 2009b) discusses PILOT science projects dealing with the nearby Universe (i.e. science themes 4-7).

\section{PILOT Design and Performance}

\subsection{Dome C Site Conditions}

Nearly two decades ago it was recognised that the high, dry, cold sites on the Antarctic plateau should be ideal for astronomy (see Storey (2005) for a recent review). Harper (1989) suggested that the low temperatures throughout the Antarctic plateau troposphere would result in a very low atmospheric thermal emission in the infrared. Townes \& Melnick (1990) suggested that the low atmospheric water vapour content would be particularly suitable for infrared and millimetre wave observations. Gillingham (1991) predicted that the calm and stable atmosphere above the Antarctic plateau would result in weak turbulence, and that the strong temperature inversion and low winds on the domes of the high plateau would result in the atmospheric turbulence being confined to a very thin but intense layer close to the surface.

Since that time, site-testing experiments have been carried out at, or are planned for, a number of locations (South Pole, Dome C, Dome A, and Dome F) on the Antarctic plateau (see Figure 1). These results have confirmed and quantified the earlier expectations, and have demonstrated that each of these sites has distinct characteristics, 
and is therefore most appropriate to a specific astronomical field. Dome C, the site of the French/Italian Concordia station (see Candidi \& Lori 2003), has been selected as the most appropriate location on the plateau for the PILOT telescope.

Dome C, at $123^{\circ}$ East longitude and $75^{\circ}$ South latitude, is at an altitude of $3250 \mathrm{~m}$. It has been operated by the French and Italian Antarctic agencies, Institut Paul Emile Victor (IPEV) and Programma Nazionale di Ricerche in Antartide (PNRA), during the summer months since 1996, and has been manned year round since 2005 .

The key atmospheric parameters and site conditions at Dome $\mathrm{C}$ relevant to the scientific potential for PILOT are ${ }^{2}$ :

- excellent free-atmospheric seeing (Lawrence et al. 2004; Agabi et al. 2006; Trinquet et al. 2008),

- low turbulent boundary layer height (Lawrence et al. 2004; Agabi et al. 2006; Trinquet et al. 2008),

- wide isoplanatic angle and long coherence time (Lawrence et al. 2004; Agabi et al. 2006; Trinquet et al. 2008),

- low atmospheric scintillation (Kenyon et al. 2006),

- low sky and telescope thermal emission (Lawrence 2004; Walden et al. 2005),

- low precipitable water vapour content (Valenziano \& Dall'Oglio 1999; Lawrence 2004; Tomasi et al. 2006),

- high cloud-free fraction (Mosser \& Aristidi 2007), and

- high latitude (Kenyon \& Storey 2006).

\subsection{PILOT Configuration}

The baseline optical design for PILOT, described in Saunders et al. (2008a) ${ }^{3}$, comprises a 2.5-m Ritchey-Chretien telescope with $f / 1.5$ primary and $f / 10$ overall focal ratios, giving diffraction-limited performance at $1 \mu \mathrm{m}$ over a $1^{\circ}$ field. Instruments are mounted on twin Nasmyth foci. The telescope is housed in a calotte-style dome that is mounted on top of a $\sim 30-\mathrm{m}$ high tower in order to elevate the main mirror above the majority of the intense ground-layer turbulence (see Figure 2). The enclosure is temperature and humidity controlled, protecting the optical elements from large spatial and temporal thermal gradients, and preventing frost formation on optical surfaces (Durand et al. 2007). A fast tip-tilt secondary mirror is used for guiding and to remove residual boundary-layer turbulence and tower wind-shake. The system is designed for continuous 24-hour remote operation with minimal human intervention.

\subsection{PILOT Baseline Instruments}

The following list shows the types of astronomical observations where PILOT can outperform a similar

\footnotetext{
${ }^{2}$ The PILOT Dome C Environmental Conditions Document (and references therein) describes in detail the meteorological atmospheric conditions and astronomical site conditions at Dome C. See http : / / www.phys. unsw. edu.au/pilot/pilot status.htm.

${ }^{3}$ See also the PILOT Functional and Performance Requirements Document at http: //www.phys.unsw.edu.au/pilot/ pilot_status.htm.
}

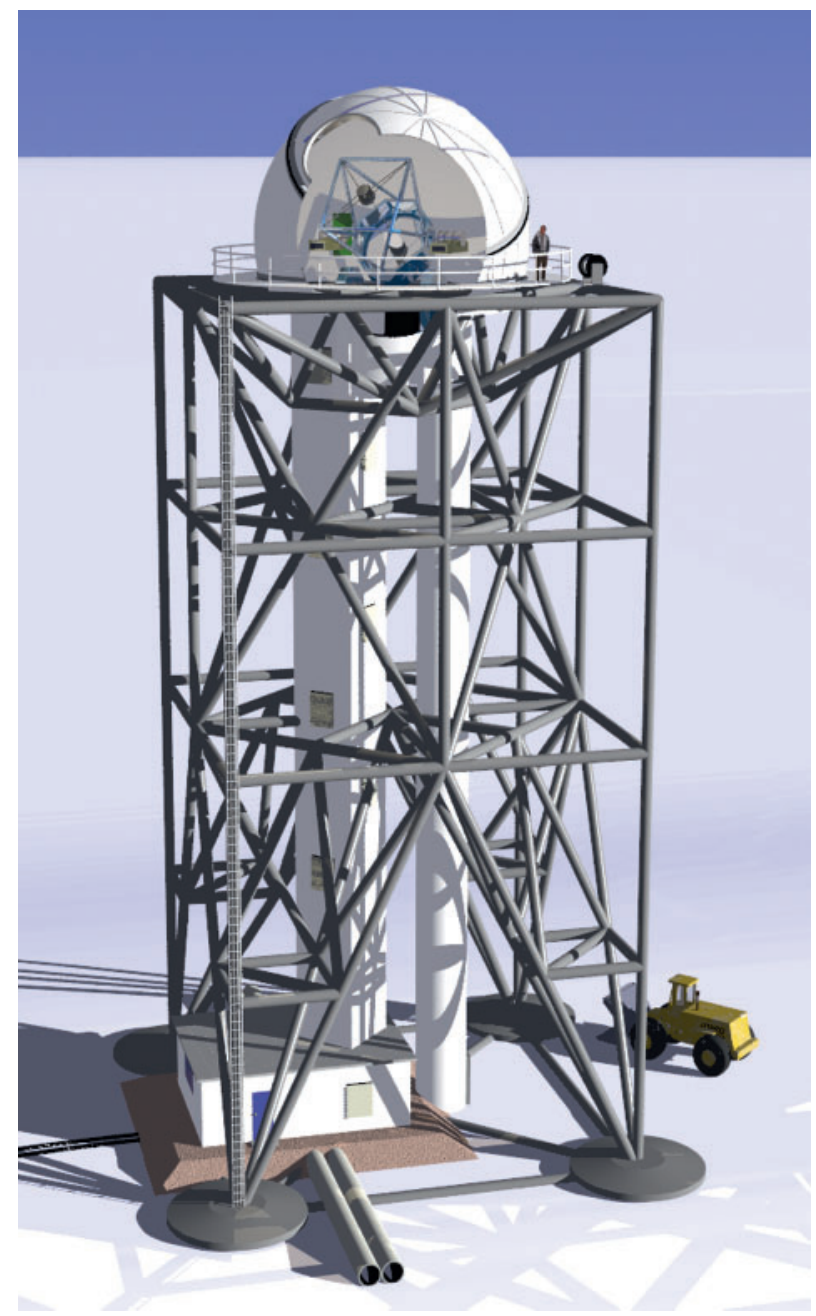

Figure 2 Concept design for the PILOT telescope, enclosure, and tower.

or somewhat larger telescope at a temperate latitude observatory.

- High-resolution near-diffraction-limited imaging in the visible over small fields (resulting from the excellent free-atmospheric seeing, the wide atmospheric isoplanatic angle, and the long atmospheric coherence time).

- Moderate-resolution wide-field imaging in the visible and near-infrared with partial (tip-tilt) correction of the residual boundary-layer turbulence (resulting from the excellent free-atmospheric seeing, the low height of the turbulent boundary layer, the wide atmospheric isoplanatic angle, and the long atmospheric coherence time), and moderate-resolution wide-field imaging in the mid-infrared (limited by the telescope aperture rather than the atmospheric conditions).

- High sensitivity in the near-infrared (arising from the low atmospheric thermal emission) and the midinfrared (arising from a combination of the low atmospheric thermal emission and the high atmospheric transmission). 
Table 1. PILOT baseline instruments, their main parameters and primary science drivers

\begin{tabular}{llccc}
\hline Instrument & Primary science & $\begin{array}{c}\text { Pix scale } \\
\left({ }^{\prime \prime} / \mathrm{px}\right)\end{array}$ & $\begin{array}{c}\lambda \text { range } \\
(\mu \mathrm{m})\end{array}$ & $\begin{array}{c}\text { FOV } \\
\end{array}$ \\
\hline PVISC: visible camera & Weak lensing (Paper II) & 0.08 & $0.4-1$ & $40^{\prime} \times 40^{\prime}$ \\
PNIRC: near-infrared camera & First light, high- $z$ gal. (Paper II) & 0.06 & $1-2.5$ & $4^{\prime} \times 4^{\prime}$ \\
& & 0.15 & $1-5$ & $10^{\prime} \times 10^{\prime}$ \\
PMIRIS: mid-infrared imaging spectrometer & Galactic ecology (Paper III) & 0.8 & $7-25$ & $14^{\prime} \times 14^{\prime}$ \\
& & 1.3 & $17-40$ & $5^{\prime} \times 5^{\prime}$ \\
PLIC: Lucky Imaging camera & 'Hubble from the Ground' (Paper I) & 0.03 & $0.4-1$ & $0.5^{\prime} \times 0.5^{\prime}$ \\
\hline
\end{tabular}

- High photometric precision in the optical (enabled by the low atmospheric scintillation) and the infrared (enabled by the stable atmospheric thermal emission).

- Continuous coverage (due to the high latitude of the Dome $\mathrm{C}$ site and the high cloud-free fraction).

The baseline instrument suite for PILOT has been designed to take advantage of all the above opportunities. The suite consists of four cameras, of which three are imaging only and one has spectro-photometric capabilities. All four instruments will be permanently mounted on the telescope Nasmyth platform in such a way that they are individually selectable via a tertiary fold-mirror.

The four instruments, described in detail by Saunders et al. (2008b), are:

- PVISC (PILOT VISible Camera): a wide-field optical camera with ground-layer tip-tilt correction.

- PNIRC (PILOT Near-InfraRed Camera): a wide-field near-infrared camera with ground-layer tip-tilt correction and adjustable pixel scales matched to the diffraction limit at short and long wavelengths.

- PMIRIS (PILOT Mid-InfraRed Imaging Spectrometer): a wide-field mid-infrared instrument with a tuneable Fabry-Perot filter or a GRISM spectrometer, and two separate arms with short and long wavelength ranges.

- PLIC (PILOT Lucky Imaging Camera): a fast optical camera for diffraction-limited imaging over relatively small fields in the visible.

The key parameters for each of these instruments, and the appropriate primary science drivers, are given in Table 1. The specifications given here should be considered indicative; a range of applicable values for each instrument specification has been identified ${ }^{4}$ and awaits a more detailed trade-off analysis before selection of the optimum configuration.

\subsection{Advanced Technology Instruments}

In addition to the baseline instruments defined in the previous section, there are several 'advanced technology' instruments that are also being considered. These are either instruments for a generic $\sim 2-\mathrm{m}$ class Antarctic telescope

\footnotetext{
${ }^{4}$ See the PILOT Science Requirements Document and the PILOT Functional and Performance Requirements Document at http:// www.phys.unsw.edu.au/pilot/pilot_status.htm.
}

that are being developed independent to the PILOT project, or instrument configurations that involve a significant extension to the baseline specifications. They include:

- SmilePILOT: a sub-millimetre imaging camera. The SmilePILOT instrument consists of a filled-bolometer array camera operating from 200 to $450 \mu \mathrm{m}$ (Minier et al. 2007, 2008). At $200 \mu \mathrm{m}$, it will provide a FWHM beam of $21^{\prime \prime}$, with a total field-of-view of $9.2^{\prime} \times 9.2^{\prime}$.

- AIFU (Antarctic Integral Field Unit): a near-infrared integral field spectrograph fed with a fibre array (Le Bertre, Epchtein \& Vauglin 2008). This instrument samples a $1^{\prime} \times 1^{\prime}$ field-of-view at $2^{\prime \prime}$, giving a spectral resolution of $R \approx 100$ covering the wavelength range $1-2.5 \mu \mathrm{m}$.

- Polar Bear: an Imaging Fourier Transform Spectrometer (IFTS) to operate in the near-infrared (Maillard \& Boulanger 2008). Polar Bear consists of a dual-arm interferometer sampling a $10^{\prime} \times 10^{\prime}$ field-of-view at $0.3^{\prime \prime}$ operating over the wavelength range $1.8-5.5 \mu \mathrm{m}$, with a maximum spectral resolution of $R=125000$.

- PSDC (PILOT Satellite Debris Camera): a wide-field imaging camera for satellite debris tracking. This instrument, which consists of a large mosaic of fast-readout CCDs, should obtain high spatial resolution imaging in the visible over a wide field $\left(1 \mathrm{deg}^{2}\right)$.

\subsection{PILOT Performance}

The design for the PILOT telescope and instrument suite must ultimately be driven by the requirements determined by the scientific objectives. These objectives can only be defined based on some initial specification. The starting point for the science case is the baseline instrument suite, described in Section 2.3, and its expected performance, described here.

The estimated system performance, i.e. achieved resolution and sensitivity, is given in Table 2, based on the analysis of Saunders et al. (2008a). The image quality specification includes telescope aberrations and surface errors; misalignment errors; telescope, mirror, and dome seeing; residual tower wind-shake; and guiding errors introduced via the tip-tilt measurement and correction system. The sensitivity analysis includes sky and telescope thermal emission, and assumes optimum source extraction. The expected resolution and sensitivity for PILOT depends on many site parameters (particularly 
Table 2. The expected resolution and sensitivity for PILOT in a number of wavebands

\begin{tabular}{lccccccc}
\hline Band & $\begin{array}{c}\lambda \\
(\mu \mathrm{m})\end{array}$ & $\begin{array}{c}R \\
(\lambda / \Delta \lambda)\end{array}$ & $\begin{array}{c}\mathrm{FWHM}^{\mathrm{a}} \\
(\operatorname{arcsec})\end{array}$ & $m_{\mathrm{AB}} \mathrm{b}^{2}$ & $m_{\mathrm{Vega}} \mathrm{b}^{2}$ & $\begin{array}{c}m_{\mathrm{AB}} \mathrm{b}^{-2} \\
\left(\operatorname{arcsec}^{-2}\right)\end{array}$ & $\begin{array}{c}m_{\text {Vega }}{ }^{\mathrm{b}} \\
\left(\operatorname{arcsec}^{-2}\right)\end{array}$ \\
\hline$g$ & 0.47 & 3.4 & 0.35 & 27.6 & 27.6 & 27.1 & 27.1 \\
$r$ & 0.62 & 4.4 & 0.33 & 27.1 & 26.9 & 26.5 & 26.3 \\
$i$ & 0.76 & 5.1 & 0.32 & 26.6 & 26.2 & 26.0 & 25.6 \\
$z$ & 0.91 & 6.5 & 0.31 & 25.8 & 25.3 & 25.1 & 24.6 \\
$Y$ & 1.04 & 5.1 & 0.30 & 25.5 & 24.9 & 24.8 & 24.2 \\
$J$ & 1.21 & 4.6 & 0.30 & 25.0 & 24.1 & 24.3 & 23.4 \\
$H$ & 1.65 & 5.7 & 0.29 & 24.6 & 23.2 & 23.8 & 22.4 \\
$K_{\mathrm{d}}$ & $2.40^{\mathrm{c}}$ & 10 & 0.32 & 25.3 & 23.3 & 24.7 & 22.7 \\
$L$ & 3.76 & 5.8 & 0.40 & 21.2 & 18.3 & 20.8 & 17.9 \\
$M$ & 4.66 & 19 & 0.46 & 19.6 & 16.2 & 19.4 & 16.0 \\
$N^{\prime}$ & 11.5 & 11 & 1.05 & 16.3 & 11.2 & 17.0 & 11.9 \\
$Q_{\mathrm{N}}$ & 20.1 & 20 & 1.80 & 14.6 & 8.1 & 15.8 & 9.3 \\
\hline
\end{tabular}

\footnotetext{
${ }^{a}$ The resolution full-width half maximum (FWHM) over the full imaging field-of-view for each camera, is given as a function of wavelength based on a preliminary analysis of the efficiency of the proposed ground-layer tip-tilt correction system. For wavelengths longer than $\sim 3 \mu \mathrm{m}$ the corrected resolution is close to diffraction limited.

${ }^{\mathrm{b}}$ Point-source and extended-object limiting sensitivities (in magnitudes and magnitudes per square arcsec respectively) are given for a 5- $\sigma, 1$-hour integration, assuming that the sky background is summed over 4 times the FHWM disc (for point sources), the telescope is at $227 \mathrm{~K}$ with $5 \%$ emissivity, the overall optical efficiency is $50 \%$ (including throughput, detector efficiencies, and secondary mirror obscuration) and the infrared sky backgrounds are as given in Burton et al. (2005).

${ }^{c}$ The $K_{\mathrm{d}}$-band (or $K_{\mathrm{dark}}$ band) is defined with a centre wavelength of $2.4 \mu \mathrm{m}$. This band is centred at a slightly higher wavelength than the standard $K$ band, in order to take maximum advantage of the low thermal emission of the Antarctic atmosphere.
}

the free-atmospheric seeing, the turbulence profile, and the thermal infrared background) that are not completely characterised, and also on details of the telescope design (e.g. plate scale, baffling, guiding system) that are not yet finalised. The estimated system performance, as summarised in Table 2, is thus intended to be indicative rather than definitive.

\subsection{PILOT Discovery Space}

The scientific capabilities for PILOT need to be considered in the context of the capabilities of other current, planned, or proposed facilities. Figure 3 shows the resolution and relative survey time (ratio of integration times required to image a given area of the sky to a given sensitivity) for PILOT compared to a number of other facilities in the visible ( $i$ band), near-infrared ( $K$ band), and mid-infrared ( $Q$ band). One of the key benefits of PILOT is that it will operate over this entire wavelength range. The relative efficiency metric used here includes, for each telescope, pixel scale, resolution (either seeing-limited or corrected using adaptive optics), telescope effective diameter, atmospheric (or space) thermal emission, telescope thermal emission, and instrument field-of-view. ${ }^{5}$ In Figure 3 we

\footnotetext{
${ }^{5}$ Telescope, instrument, and site parameters taken from Kaiser et al. (2002); Fazio et al. (2004); Rhodes et al. (2004); Rieke et al. (2004); Sholl et al. (2004); Dalton et al. (2006); Mainzer et al. (2006); Tokovinin \& Travouillon (2006); Werner et al. (2006); Keller et al. (2007); Thomas-Osip (2007); Ivezic et al. (2008) and http://www.gemini.edu/sciops/instruments/; http://www.gmto.org/CoDRpublic; http://www.eso. org/sci/facilities/paranal/instruments; http:// www. eso.org/gen-fac/pubs/astclim/paranal/
}

show both general purpose and survey telescopes. As expected, PILOT is usually (though not always) faster in a survey mode than telescopes that are not designed with surveys in mind.

In the visible, PILOT provides a higher resolution (not including Lucky Imaging systems) and a faster survey speed than any current ground-based telescope. HST (Hubble Space Telescope) provides a higher resolution, but is slower. Future wide-field optical facilities such as Pan-STARRS-4 (Panoramic Survey Telescope And Rapid Response System) and LSST (Large Synoptic Survey Telescope) are a factor 10-40 times faster than PILOT but will not achieve the same spatial resolution. PILOT is faster at imaging and has a higher resolution than ground based ELTs (Extremely Large Telescopes) in seeinglimited mode, but loses the advantage if GLAO (Ground Layer Adaptive Optic) systems can be routinely operated on those facilities. It is unlikely, however, that ELTs will do large imaging surveys, as they are better suited to narrow spectral resolution work in this wavelength range. The proposed Euclid, previously DUNE (Dark UNiverse Explorer), and SNAP (SuperNova/Acceleration Probe) space missions provide slightly better spatial resolution and are 4-12 times faster than PILOT. They are not due

\footnotetext{
seeing/; http://wise.ssl.berkeley.edu/mission. html; http://www.spitzer.caltech.edu/; http:// casu.ast.cam.ac.uk/documents/vista/; http://www. tmt.org/index.html; http://vstportal.oacn.inaf. it/node/1; http://www.stsci.edu/hst/; http://www. dune-mission.net/; http://snap.lbl.gov/; http:// www.lsst.org/lsst_home.shtml; http://pan-starrs. ifa.hawaii.edu/public/home.html.
} 

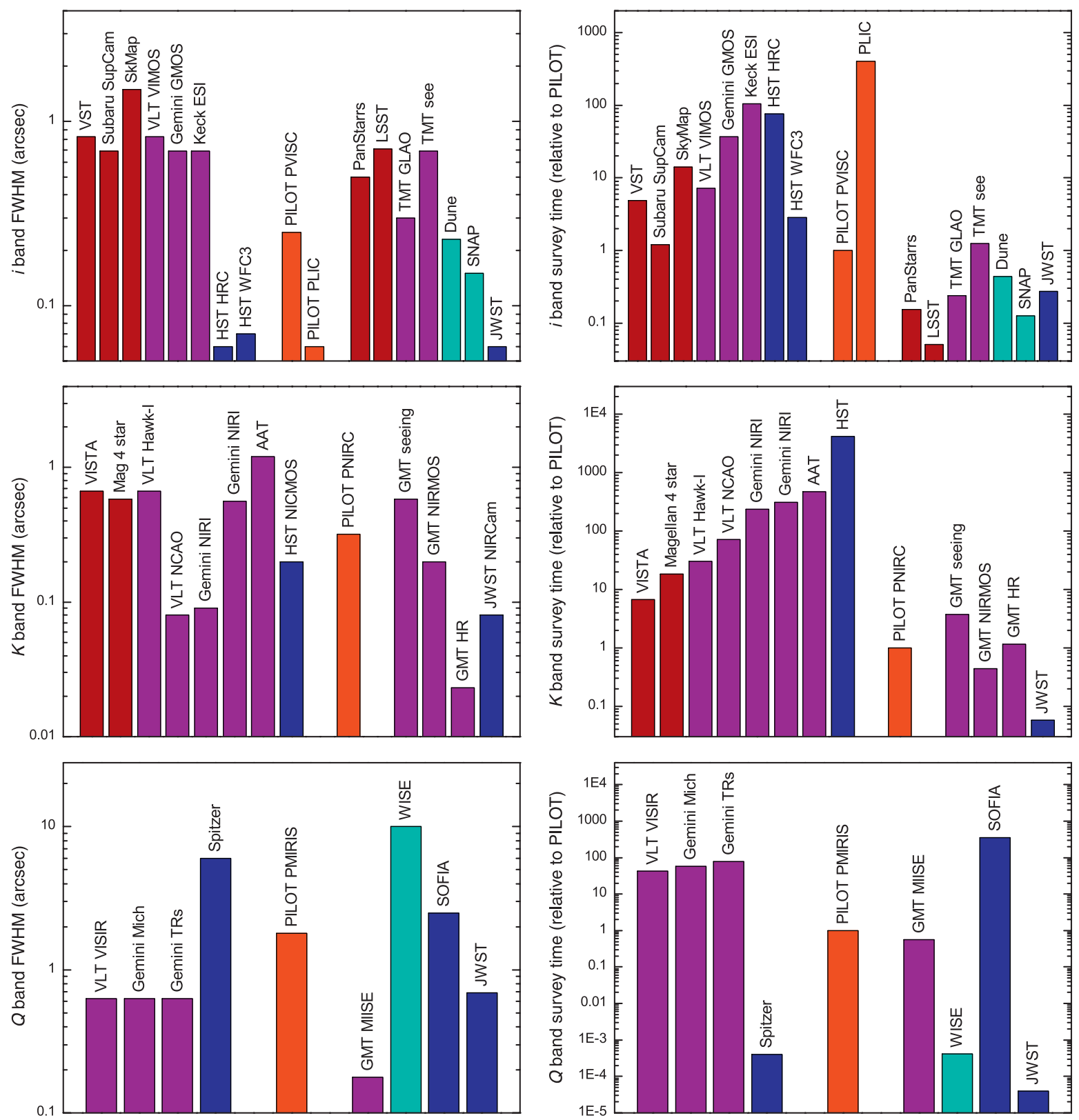

Figure 3 Resolution (left column) and survey time relative to PILOT (right column) for a range of facilities at $i$ band (top row), $K$ band (middle row), and $Q$ band (bottom row). Survey time represents the time required to survey a given area of sky to a given depth. For both FWHM and survey time a lower number is preferable (i.e. giving a high spatial resolution in a short amount of observing time). The comparison includes wide-field ground-based survey telescopes or instruments (brown), ground-based general-purpose telescopes (purple), space-based wide-field survey telescopes (light blue), and spaced-based general-purpose telescopes (dark blue). Existing and near-complete facilities are shown to the left of PILOT, and future planned or proposed facilities to the right of PILOT.

for first light until at least 5 years after PILOT; it is thus possible that PILOT could achieve many of their proposed science goals before they are launched. The planned visible camera for JWST (James Webb Space Telescope) provides a factor of $\sim 6$ in imaging speed and has a much higher spatial resolution than PILOT. However, JWST is optimised for performance in the thermal infrared and it is currently not expected that large projects in the visible will be undertaken.

The PILOT niche in the near-infrared at $K$ band arises from a combination of the relatively high spatial resolution and the low thermal background. PILOT is at least 8 times faster to image a given area of sky than any current telescope at this wavelength, and only HST and adaptive-optics equipped 8- to 10-m class telescopes can compete for resolution. PILOT is either faster with a higher resolution, or slower with a lower resolution than the GMT (Giant Magellan Telescope), depending on the GMT instrument considered (primarily related to the adaptive optics capabilities). As with the optical, however, the key ELT role in the near-infrared will be high spectral and/or spatial resolution observations. JWST will the pre-eminent telescope in the near-infrared. It is diffraction limited at these wavelengths. As shown here JWST 


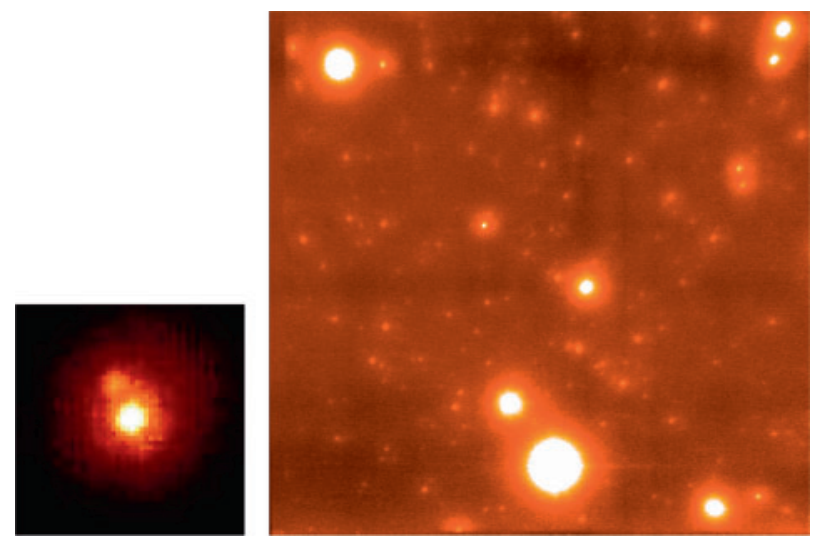

Figure 4 Left: $0.12^{\prime \prime}$ binary, $\delta_{\mathrm{mag}} \approx 2.5$, imaged with LuckyCam on the NOT $2.5-\mathrm{m}$ telescope. Right: the highest resolution optical image ever taken, with a resolution of $0.035^{\prime \prime}$ in $i$ band, using the Lucky Camera behind the Palomar 5-m telescope low-order adaptive optics system (from Mackay, Law \& Stayley 2008). A similar resolution should be achievable with PLIC on PILOT at wavelengths around $0.5 \mu \mathrm{m}$. This is $\sim 2$ times higher resolution than possible with HST.

is 10-500 times faster than any existing ground based telescope, and is $\sim 20$ times faster than PILOT at $K$ band. However, there is a significant efficiency loss associated with JWST telescope slewing and settling, which precludes its use for large-area surveys. Its real niche lies in obtaining deep high-resolution images over relatively small regions of sky.

The niche for PILOT in the mid-infrared wavelength range is clear. It falls in the large gaps between ground and space telescopes in terms of survey speed, and between large-aperture small-field telescopes and small-aperture wide-field telescopes in terms of resolution. It is the only telescope capable of obtaining moderate spatial resolution photometry over very wide regions of sky, and is thus an ideal complement to both the deep narrow fields of JWST and GMT and the wide-area low-spatial-resolution fields of Spitzer and WISE (Wide-field Infrared Survey Explorer).

\subsection{Lucky Imaging with PILOT}

The PILOT project has considerable potential to extend the parameter space demonstrated by Lucky Imaging camera systems. The unique site characteristics at Dome $\mathrm{C}$ should allow the proposed PILOT Lucky Imaging Camera (PLIC) to achieve higher resolution over a larger fraction of the sky at shorter wavelengths than other Lucky Imaging systems operated on mid-latitude telescopes. PLIC is expected to play an important 'pathfinding' role for the PILOT project, where the delivered performance will provide a confirmation of the atmospheric conditions of the site; there is no better way to verify these atmospheric conditions. The technique is therefore described in more detail here.

The Lucky Imaging technique, first proposed by Fried (1978), aims to improve the angular resolution of astronomical instruments on ground-based telescopes. The process involves the collection of sequences of short-exposure frames. The frames are then sorted by Strehl ratio. The selected fraction are then re-centred on the brightest speckle and added. Non-selected frames may be used to increase sensitivity at the expense of resolution. Lucky Imaging has been demonstrated to be routinely usable on 2.5-m class optical telescopes on good highaltitude sites and able to produce spatial resolutions of $\sim 0.1^{\prime \prime}$ in $i$ band (Law, Hodgkin \& Mackay 2008) using reference stars as faint as $m_{\mathrm{AB}}=16.3-16.8$ (Law, Mackay \& Baldwin 2006). In the near-infrared, deep depletion CCDs will soon be available which should offer an improvement in sensitivity of about one magnitude. With a suitable reference star, the faint limit for science targets is that predicted for the selected image integration time.

As the Lucky Imaging technique involves the statistics of atmospheric turbulence, the expected performance of such systems is a strong function of the atmospheric site conditions (Baldwin, Warner \& Mackay 2008). The good seeing and long coherence time of the Dome $\mathrm{C}$ atmosphere should allow the system to work with a slower frame rate, improving the reference star sensitivity. A good working estimate for the reference star limiting magnitude for PLIC is probably $m_{\mathrm{AB}} \approx 18.3$ at $i$ band, corresponding to a star density that gives a $50 \%$ chance of finding a reference star within a $1-\operatorname{arcmin}^{2}$ field at high galactic latitudes. Lucky Imaging also benefits from a large isoplanatic patch (Baldwin et al. 2008). The isoplanatic angle is typically $1^{\prime}$ at $i$ band on a site such as La Palma; the expectation is that it should be a factor 2-3 larger at Dome C. There is thus a higher probability of finding a suitable reference star within the isoplanatic patch of PILOT. In addition, ground-layer turbulence effects are negated because each frame is shifted and added before recombination. There is a significant sensitivity hit for Lucky Imaging, as the 'bad' frames are thrown away. To obtain reasonable Strehl ratios from mid-latitude sites typically requires selection of the best $1-10 \%$ of images. The number of retained images is a strong function of the atmospheric seeing, and thus, for PLIC we may expect that as many as $50 \%$ of frames can be retained, corresponding to a significant gain in sensitivity.

All of the performance metrics for Lucky Imaging (sky coverage factor, reference star limiting magnitude, number of images collected, and achieved resolution) degrade with a decrease in wavelength. PLIC should be capable of obtaining better performance than a mid-latitude Lucky Imaging system operating at the same wavelength. Alternatively, PLIC should achieve similar performance at shorter wavelengths, see Figure 4. PLIC should, in theory, be capable of delivering a higher resolution than the HST actually delivers in the $B$ and $V$ bands ${ }^{6}$.

\section{The PILOT Science Case}

\subsection{Context}

Four or five years from now, when PILOT could be operational, there will have undoubtedly been many

\footnotetext{
${ }^{6}$ See the Wide Field Camera 3 Instrument Handbook at http: // www. stsci.edu/hst/.
} 
developments in astronomy. Here we summarise the potential astronomical facility landscape at that time, assuming that currently planned projects are completed on schedule.

On the ground, a new generation of instruments will be available on current 8 - to 10-m class telescopes and multi-conjugate adaptive optics systems using multiple laser guide stars will be operating efficiently and reliably. Ground Layer Adaptive Optics (GLAO) systems on smaller-scale telescopes now being developed will have obtained useful results. The VISTA (Visible \& Infrared Survey Telescope for Astronomy), VST (VLT Survey Telescope), and Skymapper survey telescopes, after operating for several years, will have mapped large regions of the sky at optical and infrared wavelengths. Currently proposed large optical survey telescopes, LSST and Pan-STARRS-4, will be nearing completion, and one or more of the current ELT projects will be fully funded and well into their construction phase.

At longer wavelengths, the ALMA (Atacama Large Millimeter Array) sub-millimetre array will have achieved first light, a site will have been selected for the Square Kilometre Array (SKA) radio array, and SKA prototype facilities, such as the Australian Square Kilometre Array Pathfinder (ASKAP) and the Murchison Widefield Array (MWA), will be fully operational.

In space, JWST will have achieved first light, NASA will have selected the Joint Dark Energy Mission (JDEM) project from current mission concepts (SNAP, ADEPT, Destiny), and ESA will have selected a Cosmic Visions project from current candidates (such as Euclid). The HST may still be operational, and Kepler and Corot will have collected many years of data on the transient optical sky. WISE and Spitzer will have mapped significant areas of the sky in the mid-infrared.

This array of new facilities will allow new observational possibilities, opening up new areas of astronomical study. While our understanding of the Universe will thus be significantly increased, there will no doubt continue to be a vast array of unanswered questions in all fields of astronomy.

Despite the large scope of these new facilities, the capabilities of PILOT will be unique; it will provide the highest spatial resolution observations (at any wavelength) of any wide-field survey-telescope and it will operate at wavelengths from the visible to the mid-infrared. These capabilities will allow PILOT to address some of the fundamental questions in astronomy. PILOT will allow us to study the structure and composition of the first stars to form in the Universe and the environments in which they form; the history of reionisation in the Universe and the characteristics of distant galaxies and clusters; the nature and evolution of dark matter and dark energy; the internal properties of stars and the stellar populations of nearby galaxies; the molecular phase of the Galaxy and the processes of star and planet formation; the physical and chemical properties of exoplanets; and the characteristics of planetary atmospheres.
Table 3. Indicative parameters ${ }^{\mathrm{a}}$ for the main PILOT science projects

\begin{tabular}{llrc}
\hline Theme: Project & Wavebands & $\begin{array}{c}\text { Sky area } \\
\left(\mathrm{deg}^{2}\right)\end{array}$ & $\begin{array}{c}\text { Observing } \\
\text { time (hours) }\end{array}$ \\
\hline First light: & & & \\
$\quad$ PISN & $K_{\mathrm{d}}$ & 40 & 6000 \\
$\quad$ GRB & $J, H, K_{\mathrm{d}}, L, M$ & $\mathrm{ToO}$ & 200 \\
Structure: & $K_{\mathrm{d}}$ & 3 & 500 \\
$\quad$ High- $z$ galaxies & $r, i, z$ & 40 & 300 \\
$\quad$ Clusters & & & \\
Dark energy: & $r+i+z$ & 4000 & 2500 \\
$\quad$ Weak lensing & $J, H, K_{\mathrm{d}}$ & 6 & 2500 \\
$\quad$ SN Ia & $g, r, J, K_{\mathrm{d}}$ & 2 & 800 \\
Stellar pops: & $Y, J, K_{\mathrm{d}}$ & 5 & 4000 \\
$\quad$ Local Group & $L, K_{\mathrm{d}}$ & 50 & 2000 \\
$\quad$ Satellites & & 2 & 1000 \\
$\quad$ LMC & & & \\
$\quad$ Asteroseismology & $r$ & 10 & 6000 \\
Star formation: & $12,17 \mu \mathrm{m}$ & 64 & 3500 \\
$\quad \mathrm{H}_{2}$ survey & $N, Q$ & & \\
$\quad$ Chamaeleon & & 10 & 1000 \\
Exoplanets: & $J, H, K_{\mathrm{d}}$ & $\mathrm{ToO}$ & 750 \\
$\quad$ Free-floaters & $g, r$ & 1 & 500 \\
$\quad$ Microlensing & & & \\
Solar System: & & & \\
$\quad$ Planets & $g, z, K_{\mathrm{d}}$ & & \\
\hline
\end{tabular}

${ }^{a}$ Wavebands, sky coverage, and required observing time. $\mathrm{ToO}=$ Target of Opportunity single-object observations.

\subsection{Science Case Summary}

Here we summarise the key science projects for the PILOT facility. More detail is given in the companion papers in this series. Paper II addresses projects under the themes first light in the Universe, the assembly of structure, and dark matter and dark energy. Paper III addresses projects under the themes stellar properties and populations, star and planet formation, exoplanet science, and solar system and space science. In Table 3 we list indicative wavebands, sky coverage, and required observing time for the main science projects.

First light in the Universe: a near-infrared search for pair-instability supernovae (via a dedicated wide-field survey) and gamma-ray burst afterglows (via alerts from highenergy satellites), events which represent the final evolutionary stages of the first stars to form in the Universe.

The assembly of structure: a deep and wide survey in the near-infrared to study galaxy structure, formation, and evolution via the detection of a large sample of highredshift galaxies; and a study of a sample of moderateredshift galaxy clusters in the optical aimed at understanding galaxy-cluster growth, structure, and evolution.

Dark matter and dark energy: a wide-area optical survey that will probe the evolution of dark matter and dark energy via the measurement of the ellipticities of a large sample of weak gravitationally-lensed galaxies; and a near-infrared search for Type Ia supernovae to obtain light curves that are largely unaffected by dust extinction 
and reddening, allowing tighter constraints to be placed on the expansion of the Universe.

Stellar properties and populations: an optical/ near-infrared survey of disc galaxies in the Local Group to study the processes of galaxy formation and evolution; an infrared survey of nearby satellite galaxies to trace their outer morphology, structure, age, and metallicity; a deep mid-infrared survey of the Large and Small Magellanic clouds in order to understand star formation processes; and long time-series optical observations of nearby globular and open clusters to study age-metallicity relationships, test various predictions of stellar astrophysics, and improve our understanding of the physics of massive stars.

Star and planet formation: a southern Galactic Plane survey of molecular hydrogen in the mid-infrared in order to further our understanding of the ecology of star formation; and a series of mid-infrared spectrophotometric surveys searching for signatures of embedded protostars, crystalline silicates, and circumstellar discs around young stellar objects and brown dwarfs.

Exoplanet science: a near-infrared search for freefloating planetary mass objects in nearby stellar clusters; a program for optical follow-up of gravitational microlensing candidates based on alerts from dedicated survey telescope networks; and a study of high precision photometric near- and mid-infrared light curves of previously discovered exoplanets.

Solar system and space science: a series of optical and near-infrared studies aimed at characterising the composition and dynamics of planetary atmospheres of Venus and Mars; a study of the Sun at mid-infrared and sub-millimetre wavelengths aimed at understanding the physical mechanisms that are responsible for coronal mass ejections and solar flares; and a monitoring program aimed at determining orbits for a large number of small Low Earth Orbit satellite debris items.

\subsection{A PILOT Mission}

The PILOT science case is derived from a diversity of science objectives within a wide range of fields under the themes just discussed. There are several projects, however, that we have identified to represent the 'flagship' science for the PILOT facility. These four scientific projects have a realistic potential to deliver high-impact breakthrough discoveries, and have been the priority drivers for the telescope optical and instrumentation suite design:

1. Pair-instability supernovae and gamma-ray burst afterglow searches (Paper II: Sections 2.1 and 2.2);

2. High-redshift galaxy survey (Paper II: Section 3.1);

3. Weak lensing (Paper II: Section 4.1);

4. Galactic ecology (Paper III: Section 3.1).

\subsection{The Pathfinding Role}

A secondary objective for PILOT is to act as a 'pathfinder' facility. Firstly, as at all sites, it is not until a telescope large enough to exploit the scientific opportunities has been deployed that it is possible to truly understand the potential — and limitations — of the site. Secondly, PILOT should demonstrate solutions to the technological and engineering challenges that result from the extreme environmental conditions at Dome $\mathrm{C}$. These include, for example, the problem of diamond dust and frost accumulation on optical surfaces. Thirdly, PILOT should demonstrate the logistical and operational feasibility of setting up and running an optical/infrared observatory at the Dome $\mathrm{C}$ site, where access is restricted to only a few months per year, and there are limited on-site technical and support staff and facilities.

There are already several larger-scale future Dome C facilities proposed to follow PILOT that rely on PILOT having demonstrated the above pathfinder objectives (see Section 6). These include LAPCAT (Large Antarctic Plateau Clear-Aperture Telescope) an 8.4-m diameter off-axis optical/infrared telescope with a primary science driver to directly image exoplanets in the thermal infrared (Storey et al. 2006); and IRAST (InfraRed Antarctic Survey Telescope) proposed here as a large aperture, 8- to 10-m class, wide-field infrared survey telescope.

Future large-scale Antarctic facilities will rely on the successful implementation and operation of PILOT in much the same way that PILOT will rely on previous Antarctic infrared telescope projects, such as the 0.6-m South Pole InfraRed Explorer (SPIREX; Fowler et al. 1998), which was operated at the South Pole from 1994 to 1999. SPIREX was initially used for site testing, i.e. to quantify the conditions for infrared observations (Nguyen et al. 1996). It was then used with a 1- to 2.5$\mu \mathrm{m}$ imager (1994-1997; see Herald et al. 1990), before being equipped with a 2.4- to 5- $\mu \mathrm{m}$ imager from 1998-99. As summarised in Rathborne \& Burton (2005), SPIREX undertook a number of science programs, examining the incidence of disks around young stellar objects through their infrared excess, and studying the galactic ecology through extended 3.3- $\mu \mathrm{m}$ PAHs emission in star forming complexes. Despite its modest size, it obtained the deepest image in the 3.5- $\mu \mathrm{m} L$ band then obtained (in 1999), of the embedded stellar content in the 30 Doradus region of the LMC (Maercker \& Burton 2005), detecting a source as faint as $m_{\mathrm{AB}}=14.5$. This was only bettered by the $8-\mathrm{m}$ VLT in 2004.

Additionally, the International Robotic Antarctic Infrared Telescope (IRAIT; Tosti et al. 2006), which is currently being deployed to Dome $\mathrm{C}$, should be operating over the next few winter seasons. IRAIT is a $0.8-\mathrm{m}$ infrared telescope equipped with a double-arm camera, AMICA, capable of imaging in a series of broad- and narrow-band filters over the range $2-28 \mu \mathrm{m}$ with a field-of-view of $\sim 2.5^{\prime} \times 2.5^{\prime}$ in each arm (Dolci et al. 2006). The primary scientific motivation for IRAIT (Guandalini, Tosti \& Busso 2008) is to conduct wide-area surveys of dense ISM regions and star forming regions, to search for intrinsically cool objects such as Brown Dwarfs, and to perform widearea surveys to investigate mass-losing evolved (Asymptotic Giant Branch) stars. It is expected that IRAIT will 
provide valuable information relevant to many aspects of the PILOT project, including technological solutions to the problems arising from the low-temperature and high relative humidity environment; observatory operating, control, and communications requirements; science case development and planning; and observing strategies.

\subsection{Unexpected Science Outcomes}

For the majority of astronomical facilities, the initial science case for that facility has not predicted many of the key scientific outputs. Such new and unexpected scientific discoveries occur, in general, whenever technological, engineering or site-related advances lead to large increases in the observational parameter space relative to existing facilities. There are many examples of such unexpected science outcomes throughout the history of astronomy. The National Geographic Palomar Observatory Sky Survey conducted during the 1950s led to the discovery, for example, of the rich properties of galaxy clusters by Abell (1958). The 3CR catalogue of bright radio sources published by Bennet (1962) led to the quasar discovery of Schmidt (1963) and Matthews \& Sandage (1963). The Hubble Deep Field (Williams et al. 1996) was not part of the original science case for the HST, yet has been one of its most important outcomes. The Sloan Digital Sky Survey resulted in a wealth of science not originally anticipated, including the discovery of Galaxy halo streams (Yanny et al. 2003), thousands of cataclysmic variables (Szkody et al. 2006), the solar abundances of QSO absorption lines (York et al. 2006a), the strict mass metallicity relationship for galaxies (Bernardi et al. 2005), the faint end slope of the quasar luminosity function (Richards et al. 2005), and the colour segregation of hundreds of thousands of asteroids (Szabo et al. 2004). While the Spitzer science case ${ }^{7}$ proposed searches for exoplanets via direct imaging, it did not predict the possibility, as recently demonstrated (e.g. Knutson et al. 2008), of characterising exoplanet atmospheres via primary transit water vapour absorption or the secondary eclipse methods.

As with these historical examples, it is likely that some of the most exciting results to come from PILOT are not included in this science case. We can be reasonably confident of this since the unique atmospheric characteristics of the Dome $\mathrm{C}$ site, combined with a specific instrument suite designed to take advantage of these characteristics, opens a new discovery space.

\section{Observing Strategies}

\subsection{Shared Cadence}

Several of the proposed science programs for PILOT require dedicated observing time in targeted fields, e.g. gamma-ray burst follow-up from satellite alert (Paper II: Section 2.2), imaging of galaxy clusters (Paper II: Section 3.2), time-series observations of $\omega$ Cen (Paper III: Section 2.4), and exoplanet follow-up observations (Paper

\footnotetext{
${ }^{7}$ NASA JPL, 1997, http://ssc.spitzer.caltech.edu/ documents/SRD.pdf.
}

III: Section 4.3). There are many other projects, however, that have overlapping observational requirements. Adopting a shared-cadence approach to these projects, i.e. by aiming to generate a dataset that can be used to satisfy the requirements for a range of science objectives, is likely to significantly increase the scientific efficiency of the PILOT facility. This strategy is much better suited to Dome C operation than more traditional observing strategies with multiple projects and observing sequences that change on a nightly basis.

Three separate projects identified here require deep observations in near-infrared bands over wide areas of the sky: the search for pair-instability supernovae at very high redshift (Paper II: Section 2.1), the search for high-redshift galaxies (Paper II: Section 3.1), and the search for dusty $\mathrm{SNe}$ at moderate redshift (Paper II: Section 4.2). While the most appropriate target fields still need to be determined for each of these projects, it is likely that there will be some overlap in the preferred fields, which will be well away from the Galactic plane. These projects can thus be pursued with a shared cadence. This would necessarily involve some compromise on observing strategy, as the PISN search requires repeat observations with periods up to several hundred days, the $\mathrm{SN}$ Ia search requires repeat observations with a period in the range 4-14 days, and the galaxy survey does not require any repeat observations. Such compromises are likely to lower the detection rate per observing time for each project, but to increase the overall efficiency when combined.

Similarly, the star and planet formation studies (Paper III: Section 3) each have observational requirements for multi-band imaging of star forming regions throughout the mid-infrared. In particular, the Chamealeon dark clouds complex has been identified as a suitable site to search for circumstellar discs around young stellar objects and brown dwarfs (Paper III: Section 3.2), crystalline silicate signatures around similar objects (Paper III: Section 3.3), and embedded class 0/I young stellar objects (Paper III: Section 3.4). While each of these projects requires a distinct set of observing bands (and spectral resolution) there is potential for a shared observing strategy in regions such as the Chamealeon dark clouds complex. There may also be some overlap with the detailed $\mathrm{H}_{2}$ maps produced as part of the proposed Galaxy ecology project (Paper III: Section 3.1).

Additionally, it should be expected that all of the proposed large-area survey projects will contain scientific 'by-products' in their datasets; the number and significance of these by-products will be strongly dependent on the observing strategy adopted. The weak-lensing survey (Paper II: Section 4.1) in the visible, for example, will image a large region of sky over several seasons. While it will require multiple exposures for each field to reduce systematic errors in shear measurement, there is no specific requirement on time sampling. The required depth per field could be built up by sampling each field with some time delay. With a multi-epoch approach as proposed, for example, for Skymapper (Keller et al. 2007) and LSST (Ivezic 


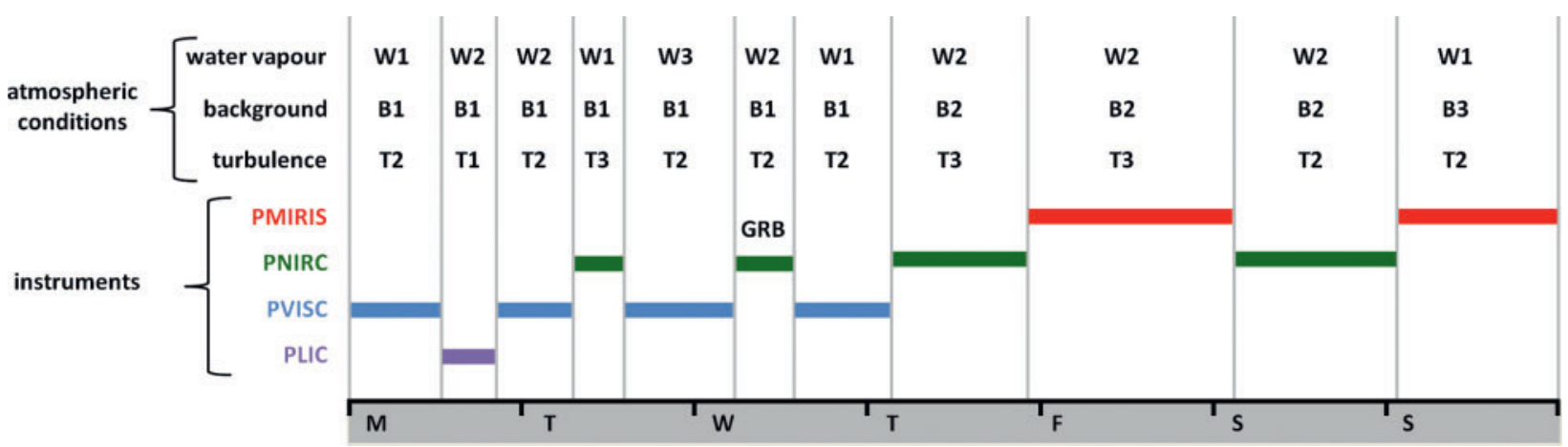

Figure 5 Illustrative observing schedule for PILOT over 1 week during mid-winter. Instruments are dynamically selected based on the atmospheric characteristics (water vapour, sky background, and turbulence conditions), and science objectives. Atmospheric conditions are ranked into a range of bands, e.g. $\mathrm{T} 1=10 \%$ best seeing, $\mathrm{B} 1=$ dark sky, etc., according to the real-time turbulent statistics, the atmospheric temperature and solar/lunar elevation, and the atmospheric precipitable water vapour content. Several science projects can override atmospheric scheduling (e.g. GRB and planet alerts).

et al. 2008), the discovery and characterisation of a large number of variable and transient objects (supernovae, solar systems objects, long- and short-period variable stars, quasars, exoplanet transits, etc.) with periods ranging from hours to years would be possible. Adopting such a strategy would necessarily increase the time required to complete the full weak-lensing survey. To decide on the most appropriate observing scheme it is thus important (for all survey projects) to identify the additional scientific output (in comparison with other proposed facilities) and to determine how this output is effected by the observing cadence chosen and hence the total observing time required.

\subsection{Dynamic Scheduling}

In combination with a shared-cadence strategy, the observational efficiency and hence scientific output of the PILOT facility would be significantly increased by employing a dynamic-scheduling approach to observing. As discussed in Section 2, there are a variety of advantages that the Dome $\mathrm{C}$ site conditions offer, such as high thermal infrared sensitivity, high photometric accuracy, and good image quality. Each science case targets these advantages in different ways and thus makes specific demands on the required observing conditions.

The efficiency of the weak-lensing survey is a strong function of the obtained imaging FWHM (full width half maximum). It is known that there are extended periods of exceptional seeing at Dome $\mathrm{C}$ that correspond either to calm conditions in the free atmosphere or a reduction of the turbulent boundary layer height to below the height of the telescope (E. Fossat, private communication). Doing this survey in only the best $50 \%$ of conditions (in terms of resolution) would be a more efficient use of facility time, than dedicating $100 \%$ of time over a full winter season. Similarly, in order to achieve the potential diffraction-limited performance with the Lucky Imaging camera, not only is the integrated seeing important, but also the atmospheric coherence time and isoplanatic angle. These factors are usually, but not necessarily, correlated with the seeing.
For optical survey projects, an efficient use of sky background conditions is essential. For example, the proposed weak-lensing survey requires dark skies, whereas the time-series observations of open clusters can be achieved in twilight conditions.

Survey programs in the near-infrared, i.e. with PNIRC, depend only weakly on the seeing (because in median conditions the resolution is close to diffraction limited) but are strongly dependent on atmospheric thermal emission (for wavelengths above $\sim 2.4 \mu \mathrm{m}$ ) and solar zenith-angle (for wavelengths below $\sim 4 \mu \mathrm{m}$ ).

Observations with the PMIRIS camera, i.e. above $\sim 7 \mu \mathrm{m}$, are essentially diffraction limited regardless of the turbulence conditions, and are insensitive to the solar zenith angle. To obtain high sensitivity at these wavelengths requires low atmospheric thermal emission. Projects requiring narrow-band observations at wavelengths corresponding to atmospheric water vapour absorption, such as proposed for the $\mathrm{H}_{2}$ mapping project, are preferentially done under conditions of low precipitable water vapour. For some projects (e.g. exoplanet secondary transits) the stability of the thermal atmospheric emission is more important than water vapour.

To satisfy these disparate demands on observing conditions will require a real-time nested scheduling strategy, where both instruments and science projects are prioritised and attributed to appropriate observing conditions. Such an observing strategy is illustrated in Figure 5 for a one week period during mid-winter. This approach adds complexity to the overall facility. Physically, a suite of real-time site monitoring instruments will be required and all telescope instruments need to be relatively easy to interchange both remotely and automatically. There will also be additional software overheads associated with such an approach. This increase in complexity (hence cost) is far outweighed, however, by the benefits in terms of total scientific output and facility efficiency.

\subsection{A Long-Term Science Program}

Is it intended that there will be several large 'flagship' science projects for the PILOT facility, and that the majority 
of observing time will be dedicated to these projects. These projects will include the four primary science mission projects described in Section 3.3. A number of other science projects will also be selected as priority science. Additionally, it is scientifically beneficial that some fraction of telescope time be devoted to smaller-scale 'queued' projects that are proposed throughout the lifetime of the facility.

While several options have been considered, the mechanism for time allocation of PILOT science projects has not yet been determined. The processes by which the science cases are prioritised, the telescope access policy, and the fraction of time available to primary, secondary, and queued projects, will be determined in the next design phase for PILOT. All of the science cases will then be further developed and refined before undergoing a selection process that will allow the observing strategy to be finalised and a motivated long-term scientific program to be developed.

During the next design phase for the PILOT project, a strategy for instrument deployment will also be determined. It is likely that a phased implementation of instruments will be employed based on a sensitivity matrix analysis of the telescope scientific functionality. One possible scenario is that PLIC will be the first instrument deployed, as it is simpler and less expensive than the other baseline instruments and it will allow a detailed characterisation of the site and telescope performance during its commissioning phase.

In Paper II and Paper III, the observational requirements for a broad range of PILOT science projects are presented. Although many of these projects require large amounts of observing time, the majority of science projects presented could be accomplished over the ten year lifetime of the PILOT facility, as illustrated in Table 3.

In Figure 6, we present an illustrative PILOT observing program for a three-year period. Observations with PMIRIS are possible in the summer months at Dome C. As shown, it should be possible to complete a molecular hydrogen survey for a wide area of the Galactic Plane in two complete observing seasons. This would allow summer-time observations in the following years to be devoted to other mid-infrared projects, such as surveys of the Chamaeleon dark clouds complex. By adopting a shared-cadence strategy, a large fraction of the proposed $K_{\mathrm{d}}$ projects (pair-instability supernovae searches, Type Ia supernovae searches, and high-redshift galaxy surveys) could be completed in a three year period. Other infrared survey projects (e.g. of the Magellanic Clouds) could then be undertaken in the following years. The weak-lensing optical project is likely to take at least five years to complete. Other smaller-scale optical projects requiring either twilight conditions (e.g. open cluster asteroseismology) or dark-sky conditions (e.g. galaxy clusters) could be completed well before then if allocated some fraction of good quality observing conditions each year. It is likely that with a full year of commissioning, the majority of PLIC projects could be completed in a few years.

\section{Synergies with Other Facilities}

The role of other facilities in contributing to PILOT science is addressed in detail in each of the science projects in Papers II and III. Here, we consider the inverse question: what value can PILOT add to the primary science from other telescopes? In particular, we consider the Giant Magellan Telescope (GMT), the South Pole Telescope (SPT), the Australian Square Kilometre Array Pathfinder (ASKAP), and the Murchison Widefield Array (MWA). This list is meant to be illustrative rather than exhaustive. For example, other large next-generation optical telescopes could be substituted for GMT.

\subsection{Giant Magellan Telescope}

The Giant Magellan Telescope is proposed as a 25-m diameter optical and infrared telescope to be sited at Las Campanas Observatory in Chile. The telescope is composed of seven 8.4-m primary mirrors and has an effective collecting area of 21.4-m. Proposed instruments include an optical high-resolution spectrometer, a near-infrared high spectral-resolution spectrometer, and a mid-infrared imaging spectrometer.

The GMT science case ${ }^{8}$ has a wide range of science drivers, many of while could benefit from PILOT observations. The GMT high resolution near- and mid-infrared sensitivities are well matched to the PILOT wide-field wide-bandwidth sensitivities, as illustrated in Figure 7.

Since the first-light for the GMT will likely occur after PILOT has achieved its primary science goals, the various PILOT wide-area surveys will be immediately useful to search for GMT infrared sources. For example, the PILOT $K_{\mathrm{d}}$-band surveys, in conjuction with photometric redshifts from Spitzer, would form an ideal high-redshift (out to $z \approx 7$ ) galaxy source catalogue for GMT. GMT could obtain high spatial and spectral resolution $(R=3000$ $100000)$ of the galaxies and use them for several key projects including the measurement of the evolution of dark energy through the exploration of baryonic oscillations, and the examination of the epoch of reionisation through the Lyman- $\alpha$ continuum break in high-redshift quasars and galaxies. In the mid-infrared, PILOT's widearea surveys will pinpoint interesting circumstellar discs for follow-up with the GMT at high spatial and spectral resolution.

After PILOT's primary science mission is completed, PILOT's main role could be as a 'finderscope' for telescopes such as GMT.

\subsection{South Pole Telescope}

The South Pole Telescope is a 10-m diameter millimetre wave telescope located at the US Amundsen-Scott South Pole station. The SPT achieved first-light in 2007 and provides a $1-$ deg $^{2}$ field-of-view with $\sim 1^{\prime}$ resolution in the frequency range $95-345 \mathrm{GHz}$. The SPT makes use of the

\footnotetext{
${ }^{8}$ http: //www.gmto.org/sciencecase.
} 

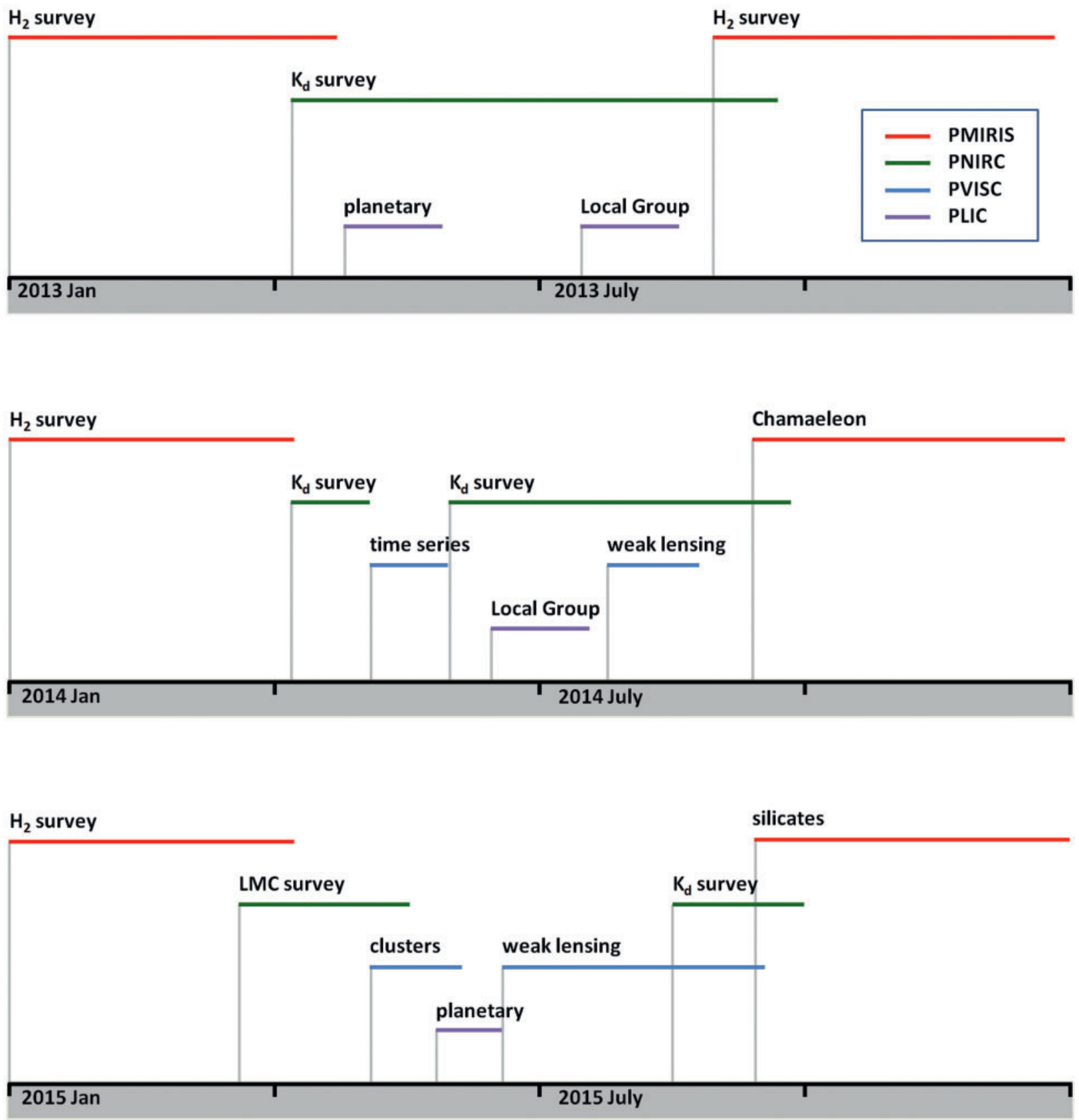

Figure 6 Illustrative observing schedule over a 3-year period for PILOT, showing the primary science project and instrument for each period. Overlapping regions represent dynamic scheduling that occurs on a much finer time-scale. It is likely that the first commissioning season (not shown here) will include extensive operation of the PLIC instrument. Here we assume that the PNIRC and PMIRIS instruments are deployed together, and that the PVISC camera is installed the following year. This illustrative scheme does not account for the time of year that objects are in appropriate locations for observation.

exceptionally low precipitable water vapour in the atmosphere above the South Pole, as well as its high temporal stability (Chamberlin 2001).

The primary science goal for the SPT is to measure the redshift evolution of the abundance of massive galaxy clusters in order to constrain cosmological parameters such as dark energy. It makes use of the SunyaevZeldovich effect (SZE) in which Cosmic microwave Background (CMB) radiation is scattered by the gas in distant galaxy clusters (see, e.g. Carlstrom, Holder \& Reese
2002). The magnitude of the SZE is directly related to the cluster mass but independent of redshift, making it an ideal cosmological probe. With a survey of $\sim 4000 \mathrm{deg}^{2}$, some 20000 galaxy clusters are expected in the redshift range $z \approx 0.1-2$ (Ruhl et al. 2004). Optical and near-infrared follow-up observations will be required for photometric redshift determination of this large sample of clusters. For objects out to $z=1.3$, redshifts will be obtained by the $(g, r, i, z)$ Dark Energy Survey (DES) on the Blanco 4-m telescope over a period of 5 years (Carlstrom et al. 2006). 


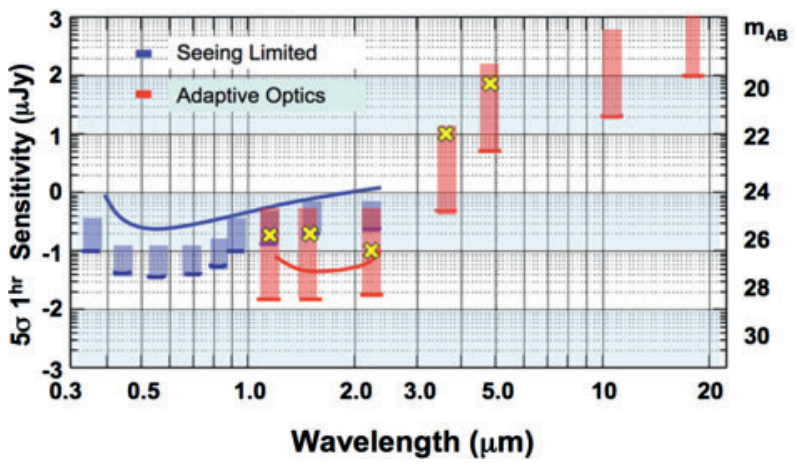

Figure 7 Sensitivity limits (for a 5- $\sigma$, 1-hour observation) for GMT. The curves show the limits for intermediate resolution spectroscopy. The shaded vertical bars show the gains over current 8-m telescopes. The top of the shaded bars are the 8-m sensitivity, the bottom is the GMT sensitivity. From the Giant Magellan Telescope Science Case. The yellow crosses show the wide-band imaging sensitivity for PILOT with PNIRC (for a 5- $\sigma$, 4-hour observation). The PNIRC field-of-view is more than 100 times the area of the diffraction-limited GMT field-of-view.

PILOT could provide an independent estimate of the masses of the SPT galaxy clusters through strong lensing events identified from surveys. The weak-lensing survey will obtain optical images of galaxies out to a redshift of $z \approx 1$ over a wide region of the sky. Such a survey would be expected to find numerous nearby galaxy clusters that are strongly lensing more distant background galaxies. Similarly, the proposed PILOT near-infrared surveys would find strongly lensed galaxy clusters at higher redshifts. The cluster masses determined from the PILOT data would be a useful verification of the SPT SZE results and a valuable test for many potential systematic effects. Additionally, PILOT near-infrared survey data could provide photometric redshifts for SZE galaxy clusters at a higher redshift $(z=1.3-2.0)$ than possible with the DES optical data.

\subsection{Australian Square Kilometre Array Pathfinder}

The Australian Square Kilometre Array Pathfinder (ASKAP) is a next generation radio telescope acting as a pathfinder for the future Square Kilometre Array (SKA). ASKAP consists of an array of 30-45 dishes of 12-m diameter observing a field-of-view of $30 \mathrm{deg}^{2}$ in the frequency range 700-1800 MHz. ASKAP is planned for deployment and commissioning in 2012. It will thus be operational throughout the lifetime of PILOT.

Key science goals for ASKAP (Johnston et al. 2007) include the detection of a statistically significant sample of low redshift $(z=0.05-0.2)$ galaxies via a $\mathrm{HI} 21-\mathrm{cm}$ survey; a confusion limited all-sky $1.4-\mathrm{GHz}$ continuum survey to detect on the order of 60 million galaxies; and the detection of a large number of radio transient events. In several of these areas, PILOT can provide a complementary role.

Although the vast majority of galaxies detected by ASKAP will be normal (Milky Way type) galaxies at low redshift $(z<0.3)$, a number of objects, such as radio galaxies (AGN) and extreme starbursts at redshifts out to $z=6$ will also be detected. Near-infrared survey data, i.e. from the Vista Hemisphere Survey (VHS) project, can be used to determine the redshift of these galaxies via the $K-z$ relationship (Jarvis et al. 2001). The depth of the VHS survey is $m_{\mathrm{AB}} \approx 22$ at $K$ band, which corresponds to a redshift of $z \approx 4$. Although there is some question about the validity of the $K-z$ relationship for high redshift (De Breuck et al. 2006), objects in the ASKAP galaxy sample that are not detected by other surveys could potentially be identified with deep $K_{\mathrm{d}}$ PILOT imaging using PNIRC.

Additionally, by virtue of its extreme southerly latitude as well as its similar longitude, PILOT is uniquely placed to rapidly follow-up any ASKAP transient event in a range of wavelengths (from the visible to the mid-infrared). Analysis of archival VLA data (Bower et al. 2007) suggests that a wide-field radio survey will find a large number of transients, many of which will be of unknown origin. Immediate searches for optical/infrared counterparts to the radio emission, and later follow-up searches for host systems, will be important steps to determine the nature of many of these objects.

\subsection{Murchison Widefield Array}

The MWA is a low frequency radio array that will be sited in the Murchison Radio Observatory in Western Australia. The array consists of 8000 dual-polarization dipole antennas operating in the $80-300 \mathrm{MHz}$ range. The majority of the tiles (each a $4 \times 4$ array of dipoles) will be concentrated within a $\sim 1.5-\mathrm{km}$ core region, allowing a resolution of a few arcmins over a field-of-view of $\sim 200 \mathrm{deg}^{2}$. The remaining tiles will be located outside this core region allowing higher angular resolution for some measurements. The MWA should be completed by the time that PILOT achieves first light.

There are three key science goals for the MWA: probing the epoch of reionisation via sensitive observations of emission and absorption in the 21-cm hyperfine transition line of neutral hydrogen that are redshifted below $\sim 200 \mathrm{MHz}$ (Morales \& Hewitt 2004); solar-heliosphericionospheric studies of solar wind parameters, crucial to the coronal mass ejections (Salah et al. 2005); and the search for low frequency transients occurring from gammaray bursts, compact objects, and stellar and planetary emission.

Each of the main science drivers for MWA will benefit from PILOT observations. The MWA observations of the cosmic neutral hydrogen over wide fields at high redshifts can be cross-correlated against PILOT measurements of the oldest and brightest distant galaxies, as illustrated in Figure 8. The MWA observations of solar wind and coronal mass ejection can be compared to lower wavelength solar observations with the proposed SmilePILOT sub-millimetre camera. Similarly to the case for ASKAP, PILOT can be used to follow-up a subset of MWA low-frequency transient sources at optical and infrared wavelengths. 


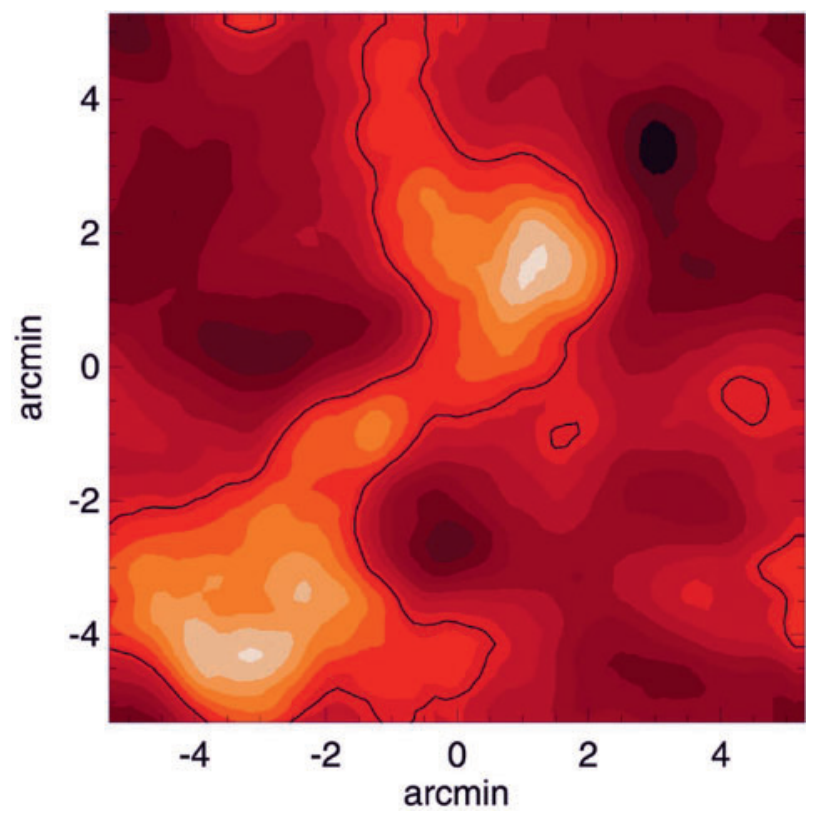

Figure 8 Simulation of redshifted 21-cm emission/absorption at $z \approx 8.5$. From Tozzi et al. (2000). The MWA aims to characterize such structure. The PILOT $K_{\mathrm{d}}$ galaxy survey would be expected to find at least four star forming galaxies, and a larger number of evolved galaxies at a redshift in the range $z=5-7$ in a field of this size.

\section{The Future of Antarctic Astronomy}

Despite its moderate sized aperture, PILOT is a powerful facility that is suited to many specific roles where it fills a favourable or even unique position in performance space, as illustrated in Section 2.6. This is also demonstrated by the significance and breadth of the scientific projects proposed in this series of papers. PILOT, however, is intended as the first step towards a large-scale astronomical observatory at Dome $\mathrm{C}$. We therefore discuss here what the future may hold.

While an ELT scale telescope at Dome C, as proposed by Angel, Lawrence \& Storey (2004), would be exceptionally powerful for a wide range of science, it is not likely to be built within the next 20 years. Here we concentrate on the facility to follow PILOT on a shorter timescale. A range of projects have been proposed that take advantage of the Dome $\mathrm{C}$ conditions to achieve a spatial resolution (using high-order or ground-layer adaptive optics systems) that is $2-3$ times higher, a near-infrared sensitivity that is 2 magnitudes deeper, and a photometric precision that is 3-4 times more accurate, than any temperate observatory. Options for this next-generation Dome $\mathrm{C}$ facility, that could conceivably be operational in the time-frame of the next $10-15$ years, include:

- LAPCAT, Large Antarctic Plateau Clear Aperture Telescope: an 8.4-m diameter off-axis telescope operating in the optical and infrared, with a highly efficient adaptive optic system (Lawrence et al. 2008) optimised for high-resolution imaging of exoplanets (Storey et al. 2006);
- IRAST, InfraRed Antarctic Survey Telescope: an 810-m class infrared telescope with a ground layer adaptive optics system operating over a very wide fieldof-view, dedicated to deep infrared survey projects that probe the first galaxies and stellar populations to form;

- ASO, Antarctic Sub-millimetre Observatory: a 12-m telescope operating at far-infrared and sub-millimetre wavelengths, with a range of science goals including the search for high-redshift optically-obscured starburst galaxies and the molecular characterisation of the interstellar medium (Olmi et al. 2005);

- Xian: a 400 element array of 0.5-m wide-field ( $\sim 20 \mathrm{deg}^{2}$ ) Schmidt telescopes which are observing a large fraction of the sky simultaneously in order to search for optical transients (York et al. 2006b);

- ALADDIN, Antarctica L-band Astrophysics Discovery Demonstrator for Interferometric Nulling: an infrared interferometer consisting of two 1-m diameter telescopes mounted on a 40-m diameter circular structure, for the characterisation of the zodiacal light for future space interferometer missions (Coudé du Foresto et al. 2005).

- KEOPS, Kiloparsec Explorer for Optical Planet Search: a 36 element interferometric array of $1.5-\mathrm{m}$ off-axis telescopes, dedicated to the detection and characterisation of exoplanets via infrared nulling (Vakili et al. 2005);

- WHAT: a LAMOST style, 8-m diameter aperture, wide-field, fixed-axis, all-reflective Schmidt telescope operating in the optical and near-infrared, suited to a range of science from galaxy evolution to large scale structure to stellar population studies of nearby galaxies (Saunders \& McGrath 2003).

\section{Conclusion}

The advantageous atmospheric and ground-level site conditions found at Dome $\mathrm{C}$ on the Antarctic plateau, combined with an optimised optical configuration and instrumentation suite designed for these conditions, allow PILOT to compete with or outperform larger-aperture telescopes located elsewhere, over a range of wavelengths. In the visible, the wide-field mode for PILOT has a spatial resolution that is $2-3$ times higher than other existing or planned ground-based telescopes, and a survey speed that is faster than any existing ground- or space-based facility. In high-resolution mode, using a Lucky Imaging system at visible wavelengths, PILOT should achieve a spatial resolution approaching the diffraction limit. In the nearinfrared, PILOT has a survey speed that is substantially faster than any existing telescope. In the mid-infrared, it is the only telescope capable of obtaining moderate spatial resolution photometry over very wide regions of sky.

A series of science projects have been identified that take advantage of the unique performance space for this telescope. These projects, which have been summarised in this paper, are described in more detail in the two companion papers in this series. The questions that can be 
addressed with PILOT cover a wide range of astronomical topics examining the Universe at a range of distance scales: from studies of the the first stars that form at very high redshift, to studies of the large-scale structure of the Universe at moderate redshift, to studies of nearby galaxies and stellar clusters, to studies of star and planet formation in the Milky Way, to studies of the Sun and planets within the Solar System. The discovery space for PILOT will also allow it to complement the scientific objectives of a variety of other existing and planned facilities operating over a broad spectral range: at visible and infrared wavelengths (e.g. GMT), at millimetre wavelengths (e.g. SPT), at radio frequencies (e.g. ASKAP), and at low frequencies (e.g. MWA).

A second objective for the PILOT project is to act as a pathfinder for future, more ambitious Antarctic optical/ infrared telescopes. In this role, PILOT must demonstrate that the Dome $\mathrm{C}$ site conditions can be fully utilised, and prove the technical and logistical feasibility of operating a large optical/infrared telescope in the Antarctic plateau environment. It must be recognised that to take full advantage of the Dome $\mathrm{C}$ site conditions will require a larger aperture $8-10 \mathrm{~m}$ class telescope; such a facility would be an exceptional tool for astronomy.

\section{Acknowledgments}

The PILOT Science Case, presented here, was produced as part of the PILOT conceptual design study, funded through the Australian Department of Education, Science, and Training through the National Collaborative Research Infrastructure Strategy (NCRIS) scheme, and the University of New South Wales through the UNSW PILOT Science Office. The European contribution has been supported by the ARENA network of the European Commission FP6 under contract RICA26150. We thank Eric Fossat and Giles Durand for providing us with data prior to publication.

\section{References}

Abell, G. O., 1958, ApJS, 3, 211

Agabi, A. et al., 2006, PASP, 118, 344

Angel, J. R. P., Lawrence, J. S. \& Storey, J. W. V., 2004, SPIE, 5382,75

Baldwin, J. E., Warner, P. J. \& Mackay, C. D., 2008, A\&A, 480, 589 Bennett, A. S., 1962, MmRAS, 68, 163

Bernardi, M., Sheth, R. K., Nichol, R. C., Schneider, D. P. \& Brinkmann, J., 2005, AJ, 129, 61

Bower, G. et al., 2007, ApJ, 666, 346

Burton, M. G. et al., 1994, PASA, 11, 127

Burton, M. G., Storey, J. W. V. \& Ashley, M. C. B., 2001, PASA, 18,158

Burton, M. G. et al., 2005, PASA, 22, 199

Candidi, M. \& Lori, A., 2003, MmSAI, 74, 29

Carlstrom, J. E., Holder, G. P. \& Reese, E. D., 2002, ARA\&A, 40,643

Carlstrom, J. E. et al., 2006, SPT White paper for the Dark Energy Task Force

Chamberlin, R. A., 2001, JGR, 106, 20101

Coudé du Foresto, V., Absil, O., Barillot, M. \& Swain, M., 2005, Proc. IAU, 1, 305
Dalton, G. B. et al., 2006, SPIE, 62694A

De Breuck et al., 2006, MNRAS, 366, 58

Dolci, M. et al., 2006, SPIE, 6267, 48

Durand, G. et al., 2007, EAS, 25, 77

Fazio, G. G. et al., 2004, ApJS, 154, 10

Fowler, A. M. et al., 1998, SPIE, 3354, 1170

Fried, D. L., 1978, JOSA, 68, 1651

Gillingham, P. R., 1991, PASA, 9, 55

Guandalini, R., Tosti, G. \& Busso, M., 2008, EAS, 33, 243

Harper, D. A., 1989, AIPC, 198, 123

Hereld, M., Harper, D. A., Pernic, R. \& Rauscher, B. J., 1990, SPIE, 1235,43

Ivezic, Z. et al., 2008, preprint (astro-ph/0805.2366)

Jarvis, M. J., Rawlings, S., Eales, S., Blundell, K. M., Bunker, A. J., Croft, S., McLure, R. J. \& Willot, C. J., 2001, MNRAS, 326, 1585

Johnston, S. et al., 2007, PASA, 24, 174

Kaiser, N. et al., 2002, SPIE, 4836, 154

Keller, S. C. et al., 2007, PASA, 24, 1

Kenyon, S. L. \& Storey, J. W. V., 2006, PASP, 118, 489

Kenyon, S. L., Lawrence, J. S., Ashley, M. C. B., Storey, J. W. V., Tokovinin, A. \& Fossat, E., 2006, PASP, 118, 924

Knutson, H. A., Charbonneau, D., Allen, L. E., Burrows, A. \& Megeath, S. T., 2008, ApJ, 673, 526

Law, N. M., Mackay, C. D. \& Baldwin, J. E., 2006, A\&A, 446, 739

Law, N. M., Hodgkin, S. T. \& Mackay, C. D., 2008, MNRAS, 384,150

Lawrence, J. S., 2004, PASP, 116, 482

Lawrence, J. S., Ashley, M. C. B., Travouillon, T. \& Tokovinin, A., 2004, Natur, 431, 278

Lawrence, J. S., Ashley, M. C. B., Storey, J. W. V., Jolissaint, L. \& Travouillon, T., 2008, PASP, 120, 1119

Lawrence, J. S. et al., 2009a, PASA, 26, 397

Lawrence, J. S. et al., 2009b, PASA, 26, 415

Le Bertre, T., Epchtein, N. \& Vauglin, I., 2008, EAS, 33, 253

Mackay, C., Law, N. \& Stayley, T. D., 2008, SPIE, 7014, 45

Maercker, M. \& Burton, M. G., 2005, A\&A, 438, 663

Maillard, J.-P. \& Boulanger, F., 2008, EAS, 33, 123

Mainzer, A. K., Eisenhardt, P., Wright, E. L., Liu, F., Irace, W., Heinrichsen, I., Cutri, R. \& Duval, V., 2006, SPIE, 6265, 61

Matthews, T. A. \& Sandage, A. R., 1963, ApJ, 138, 30

Minier V., Durand, G., Lagage, P.-O., Talvard, M., Travouillon, T. Busso, M. \& Tosti, G., 2007, EAS, 25, 321

Minier, V. et al., 2008, EAS, 33, 21

Morales, M. F. \& Hewitt, J., 2004, ApJ, 615, 7

Mosser, B. \& Aristidi, E., 2007, PASP, 119, 127

Nguyen, H. T., Rauscher, B. J., Severson, S. A., Hereld, M., Harper, D. A., Lowenstein, R. F., Morozek, F. \& Pernic, R. J., 1996, PASP, 108, 718

Olmi, L., Pelosi, G., Piccirillo, L., Saraceno, P., Sironi, G. \& Storey, J. W. V., 2005, ASPC, 14, 219

Rathborne, J. M. \& Burton, M. G., 2005, HiA, 13, 937

Rhodes, J. et al., 2004, APh, 20, 377

Richards, G. T. et al., 2005, MNRAS, 360, 839

Rieke, G. H. et al., 2004, ApJS, 154, 25

Ruhl, J. et al., 2004, SPIE, 5498, 11

Salah, J. E., Lonsdale, C. J., Oberoi, D., Cappallo, R. J. \& Kasper, J., 2005, SPIE, 5901, 124

Saunders, W. \& McGrath, A., 2003, ASPC, 13, 962

Saunders, W., Gillingham, P., McGrath, A., Haynes, R., Brzeski, J., Storey, J. \& Lawrence, J., 2008a, SPIE, 70124F

Saunders, W., Gillingham, P., McGrath, A., Haynes, R., Storey, J., Lawrence, J., Burton, M. \& Mora, A., 2008b, SPIE, 70144N

Schmidt, M., 1963, Natur, 197, 1040

Sholl, M. J. et al., 2004, SPIE, 5487, 1473

Storey, J. W. V., 2005, Antarctic Sci., 17, 555

Storey, J. W. V., Angel, J. R. P., Ashley, M. C. B., Burton, M. G., Hinz, P. M. \& Lawrence, J. S., 2006, SPIE, 6267, 45

Szabó, G., Ivezic, Z., Juric, M., Lupton, R. H. \& Kiss, L. L., 2004, MNRAS, 348, 987 
Szkody, P. et al., 2006, ApJ, 131, 973

Thomas-Osip, J. E., 2007, RMxAA, 31, 18

Tokovinin, A. \& Travouillon, T., 2006, MNRAS, 365, 1235

Tosti, G. et al., 2006, SPIE, 6267, 47

Townes, C. H. \& Melnick, G., 1990, PASP, 102, 357

Tozzi, P., Madau, P., Meiksin, A. \& Rees, M. J., 2000, ApJ, 528, 597

Tomasi, C. et al., 2006, JGR, 111, D20305

Trinquet, H., Agabi, A., Vernin, J., Azouit, M., Aristidi, E. \& Fossat, E., 2008, PASP, 120, 203
Vakili, F. et al., 2005, EAS, 14, 211

Valenziano, L. \& Dall'Oglio, G., 1999, PASA, 16, 167

Walden, V. P., Town, M. S., Halter, B. \& Storey, J. W. V., 2005, PASP, 117,300

Werner, M. et al., 2006, ARA\&A, 44, 269

Williams, R. E. et al., 1996, AJ, 112, 1335

Yanny, B. et al., 2003, ApJ, 588, 824

York, D. G. et al., 2006a, MNRAS, 367, 945

York, D. G. et al., 2006b, SPIE, 62671F 